\title{
Interkulturelle Kompetenz - Training ohne prozessbegleitendes Coaching?
}

\author{
Petia Genkova $(\mathbb{D}) \cdot$ Amsy Whiting
}

Eingegangen: 8. April 2020 / Überarbeitet: 19. Juni 2020 / Angenommen: 10. August 2020 / Online publiziert: 3. September 2020

(C) Der/die Autor(en) 2020

Zusammenfassung Durch die Globalisierung wächst der Bedarf an interkultureller Kompetenz. Diese Studie untersucht den Nutzen interkultureller Trainings, wenn diese nicht von einem Coaching ergänzt werden. In einem Querschnittsdesign wurde untersucht, inwiefern sich die interkulturelle Kompetenz von Personen mit interkulturellem Training von jenen ohne interkulturelles Training unterscheidet. Die Ergebnisse zeigen, dass interkulturelle Trainings positive Auswirkungen auf verschiedene Aspekte der interkulturellen Kompetenz haben, wobei jüngere Personen stärker von Trainings profitierten. Nachhaltige interkulturelle Handlungskompetenz kann durch ein Training allein jedoch nicht erreicht werden. Trainings sind vor allem als Vorbereitung und Ausgangspunkt geeignet.

Schlüsselwörter Interkulturelles Training · Interkulturelle Kompetenz · Ausländerfeindlichkeit · Alter

\section{The usefulness of intercultural training without in-process coaching}

Abstract Due to the ongoing process of globalization, the need for trainings for intercultural competences increases. The study aims to measure the meaningfulness of intercultural training without additional coaching. Therefore, it assessed the level of intercultural competence of participants with and without an intercultural training in a cross-sectional study design. The results show that trainings actually have a positive effect on intercultural competence. Thereby, younger participants profit more from trainings. However, results reveal that trainings are not the appropriate tool to increase actual intercultural competence, but serve as preparation measures.

P. Genkova $(\triangle)$

Caprivistr. 30A, 49076 Osnabrück, Deutschland

E-Mail: petia@genkova.de 
Keywords Intercultural Training $\cdot$ Intercultural Competence $\cdot$ Xenophobia $\cdot$ Age

\section{Einführung}

Die Internationalisierung der Arbeitswelt führt durch Auslandsaufenthalte oder multikulturelle Teams zu einer Intensivierung der Kontakte von Menschen unterschiedlicher Kulturen. Dies kann Konflikte und Probleme mit sich bringen, wie beispielsweise Rozkwitalska et al. (2017) in einer qualitativen Studie über die Belegschaft verschiedener internationaler Unternehmen in Polen untersuchten. Sie zeigen verschiedene Quellen von Problemen auf, die sie auf Unzulänglichkeiten beim Einnehmen einer fremdkulturellen Perspektive zurückführen, sowie auf mangelnde Unterstützung seitens des Managements und eine nicht förderliche Unternehmenskultur. Um derartige Probleme zu umgehen, ist es wichtig, sich mit Fragen des interkulturellen Kontextes auseinanderzusetzen und sich dadurch bestimmte, erlernbare Qualifikationen anzueignen, die unter dem Begriff interkulturelle Kompetenz subsummiert werden (Barmeyer 2010). „Interkulturelle Kompetenz“ ist definiert als die Fähigkeit in interkulturellen Situationen effizient und angemessen zu handeln (Genkova 2019). Die Kombination unterschiedlicher Sichtweisen und Arbeitsstile in kulturell diversen Teams birgt über ein problemloses Arbeiten hinaus großes Potential durch die Nutzbarmachung verschiedener Perspektiven und Stärken, wie Stegmann (2011) in einer Metastudie zeigen konnte. In kulturell diversen Teams gilt interkulturelle Kompetenz als entscheidender Erfolgsfaktor (Barmeyer 2010). Trotz inzwischen überwältigender wissenschaftlicher Evidenz für die Relevanz interkultureller Kompetenz ist die Annahme, dass kulturelle Unterschiede im Arbeitsleben eine vernachlässigbar geringe Rolle spielen, innerhalb von Organisationen weit verbreitet, berichtet Bittner (2008) in einer wissenschaftlich reflektierten Zusammenfassung der eigenen Erfahrungen als interkultureller Trainer. Zudem wird häufig bezweifelt, dass ein interkulturelles Training überhaupt einen ,lohnenden Beitrag zum Erwerb der entsprechenden Kompetenz leisten kann" (Bittner 2003). Daraus ist zu schließen, dass die generelle Wirksamkeit der interkulturellen Personalentwicklung infrage gestellt wird.

Methoden der interkulturellen Personalentwicklung werden laut Genkova (2019) differenziert in „Coaching“ einerseits und „Training“ andererseits. Während die Zielsetzung der Methoden identisch ist (möglichst guter Transfer von interkultureller Kompetenz), funktionieren sie doch deutlich unterschiedlich. Um den größtmöglichen Effekt zu erzielen, wird allgemein empfohlen, die beiden Methoden gemeinsam anzuwenden (Bittner 2008). Dies ist intuitiv nachvollziehbar, basiert jedoch mehr auf theoretischen Annahmen und praktischer Erfahrung als auf quasi-experimentellen Studien, die zur Beurteilung der Wirkung einer Maßnahme erforderlich sind. Dafür müssen die einzelnen Trainingsmethoden jeweils in ihrer Wirkungsweise untersucht werden, wie Neugebauer et al. (2019) in einem sehr ausführlichen Bericht über die Wirksamkeit von Trainings- und Coaching-Maßnahmen im Studienkontext schlussfolgern. In dieser Arbeit wird daher der Nutzen bzw. die Sinnhaftigkeit von interkulturellen Trainings ohne prozessbegleitendes Coaching betrachtet und vor dem Hintergrund praktischer Implikationen und weiterer Fortbildungsalternativen 
diskutiert. Dies trägt zum Verständnis der Möglichkeiten interkultureller Trainings bei und zeigt andersherum auf, welche Aspekte möglicherweise nicht durch ein Training ohne begleitendes Coaching abgedeckt werden können.

\section{Theoretische Grundlagen}

\subsection{Interkulturelle Kompetenz}

Treten zwei Personen unterschiedlicher Kulturen in Kontakt miteinander und tauschen sich aus, entsteht eine interkulturelle Kommunikationssituation. Die interkulturelle Kommunikation wird dabei als Austausch- und Interaktionsprozess zwischen Personen oder Gruppen mit unterschiedlichen kulturellen Hintergründen verstanden. Dabei findet nicht nur eine gegenseitige Beeinflussung der Personen statt, sondern es kann auch aufgrund unterschiedlicher Erwartungen und Bedeutungshintergründe zu Fehlinterpretationen kommen, welche die Verständigung erschweren (Barmeyer 2010). Wie bereits beschrieben, subsummiert man die Fähigkeiten, die zur Vermeidung solcher Missverständnisse beitragen, unter dem Begriff interkulturelle Kompetenz (Genkova 2019). Um sich in interkulturellen Situationen effizient und angemessen verhalten zu können, sind Perspektivenwechsel, Empathie, Ambiguität und kulturelle Sensibilität nötig (Genkova 2019; Barmeyer 2010).

Im Verlauf der jüngeren Geschichte der Psychologie sind mehrere Versuche unternommen worden, interkulturelle Kompetenz zu operationalisieren. Bolten (2006) beschreibt in einer Zusammenfassung aktueller Erkenntnisse Listen-, Struktur- und Prozessmodelle. Listenmodelle stellen oft rein additive Merkmalsauflistungen dar, bspw. „Empathie“ oder „Ambiguitätstoleranz“, die für interkulturelle Interaktion nützlich sein könnten. Strukturmodelle etablierten sich in den 1990er Jahren und unterteilen Kompetenz in affektive, kognitive und konative bzw. verhaltensbezogene Teilkonstrukte. Nach Phinney et al. (1999) kann interkulturelle Kompetenz erfasst werden als positive affektive Einstellung zur Interaktion mit anderen Kulturen. Dies umfasst die Entwicklung einer unvoreingenommenen Wahrnehmung der kulturellen Unterschiede und Ähnlichkeiten. Die kognitive Ebene beschreibt die Auseinandersetzung mit eigenen und fremden kulturbedingten Konzepten. Und drittens die verhaltensbezogene Dimension, die sich in einem effizienten und angemessenen Verhalten wiederspiegelt. Dazu zählt auch der Ausbau der Kommunikationsfähigkeit (sowohl in der eigenen, als auch in der fremden verbalen, para- und non-verbalen Sprache). Kiechl (1997) beschreibt diese als Lernzielebenen für interkulturelle Trainings. Prozessmodelle nehmen das synergetische Wechselspiel und die interdependenten Zusammenhänge zwischen Teilkompetenzen, wie Personal- bzw. Selbstkompetenz, Sozialkompetenz, Fachkompetenz und Methodenkompetenz in den Fokus. Die allgemeine eigenkulturelle Handlungskompetenz wird dabei von der fremdkulturellen Handlungskompetenz hinsichtlich des Konstrukt-Transfers auf den interkulturellen Kontext unterschieden. Ein Individuum mit interkultureller Kompetenz kann also das Zusammenspiel von individuellem, sozialem, fachlichem und strategischem Handeln in interkulturellen Kontexten erfolgreich meistern (Bolten 2007). Für Lernprozesse und Trainings zur Steigerung der interkulturellen Kompetenz werden in 
dieser Studie drei Hauptaspekte, die an das Konzept der Multicultural Personality von Van der Zee und Van Oudenhoven (2000) angelehnt sind, betrachtet. Diese sind Offenheit und Toleranz gegenüber Neuem, Einfühlungsvermögen, selbst bei scheinbar abstoßendem Verhalten des Gegenübers, und Flexibilität, das eigene Verhalten anzupassen und dies als Chance zur Persönlichkeitsentfaltung zu betrachten (Van der Zee und Van Oudenhoven 2000)

Geeraert und Demes (2012) zeigten anhand einer sowohl quer- als auch längsschnittlich angelegten Studie $(N=2500$ Studierende), dass es sich bei interkultureller Kompetenz sowohl um eine erlern- als auch eine vermittelbare Fähigkeit handelt. Die interkulturelle Kompetenz stieg somit im Zeitverlauf durch Intergruppenkontakt sowie durch Förder- und Vorbereitungsmaßnahmen. Interkulturelle Kompetenz als multidimensionales Konstrukt ist also sowohl erlern- als auch vermittelbar.

Betrachten wir interkulturelle Kompetenz als Schlüsselqualifikation auch für Personen, die nicht ins Ausland gehen, aufgrund von starker wirtschaftlicher Vernetzung und Migrationsprozessen im Arbeitsumfeld, ist eine relevante Sub-Dimension der affektiven Ebene die Einstellung zu Immigranten. Van Dick und Stegmann (2016) beschreiben in einer Zusammenfassung der aktuellen Literatur, dass die affektiven Einstellungen gegenüber der Vielfalt von bestimmten Merkmalen (z. B. kultureller Hintergrund) ausschlaggebend für das Verhalten und vor allem für die Leistungsfähigkeit von diversen Gruppen sind. Verschiedene spezielle Trainingsmethoden, zum Beispiel imaginierter Kontakt (Crisp und Turner 2012), zielen auf die Reduktion von negativen Einstellungen zu marginalisierten Gruppen. Ausländerfeindlichkeit ist also ebenfalls ein Konstrukt, das durch Weiterbildungsmaßnahmen beeinflusst werden kann. Um die Sinnhaftigkeit interkultureller Trainings zu verstehen, stellt sich die Frage, wie interkulturelle Weiterbildungskonzepte die interkulturelle Kompetenz steigern oder unterstützen und welche Stärken und Schwächen sie haben.

\subsection{Die Weiterbildungskonzepte Training \& Coaching}

Die Kernfrage bezüglich der Gestaltung interkulturellen Lernens lautet: Wie können Mitarbeiterinnen und Mitarbeiter möglichst schnell, viel, mühelos, effizient und kostengünstig lernen und dies auch nachhaltig im Beruf anwenden (Regnet und Hofmann 2003)? Zhang und Zhou (2019) unterscheiden dabei basierend auf einer Metastudie über einen Zeitraum von 18 Jahren hauptsächlich zwei Optionen, um interkulturelle Kompetenz bei Studierenden zu steigern, Auslandsaufenthalte und pädagogische Maßnahmen respektive Weiterbildungsmaßnahmen. Die zweite Option bezieht sich primär auf die Konzepte, die unter dem Begriff „Training“ verstanden werden. Die Struktur von Trainings ist auf die isolierte Förderung von Ziel- und interkulturellen Einzelkompetenzen ausgerichtet (Bolten 2001). Es handelt sich dabei im wirtschaftlichen Kontext um konventionelle kognitive und sensitive Maßnahmen off-the-job ${ }^{1}$, wobei nach Thomas und Simon (2007) verschiedene Konzepte eingesetzt werden können. Neben den von Zhang und Zhou berücksichtigten Optionen, die Universitäten anbieten, beschreibt Coaching ein Weiterbildungskon-

\footnotetext{
1 Maßnahmen off-the-job sind Fortbildungen, die sich außerhalb des gegenwärtigen oder zukünftigen Aufgabenbereichs vollziehen, oft auch außerhalb des Betriebs.
} 
zept $o n$-the-job, bei dem eine Mitarbeiterin oder ein Mitarbeiter von einem externen Coach für einen vorab festgelegten Zeitraum im Hinblick auf bestimmte Problemfelder, z. B. die der interkulturellen Interaktion, begleitet und unterstützt wird (Bolten 2001). Nichtsdestotrotz haben interkulturelle Trainings und Coachings sehr ähnliche Zielsetzungen. Nach Bittner (2003) vermittelt sowohl ein interkulturelles Training als auch ein Coaching ,den Mitarbeitern diejenigen fremdkulturellen Konzepte gezielt [...], die der Mitarbeiter kennen muss, um die Aktionen seiner fremdkulturellen Partner richtig zu verstehen und seine eigenen Aktionen so erfolgreich auf die Erwartungen der Partner abzustimmen“. Ergänzend nennt Barmeyer (2002) als Ziel des Coachings, dass „Kulturunterschiede nicht nur als Ursache von Missverständnissen und Schwierigkeiten gesehen [werden sollen], sondern als Chance für effektive und komplementäre Zusammenarbeit." Jedoch unterscheidet sich die Art und Weise, wie dieses gemeinsame Ziel erreicht werden soll.

Um die Sinnhaftigkeit interkultureller Trainings ohne prozessbegleitendes Coaching zu beleuchten, wird sich diese Arbeit im Folgenden auf Trainings und deren Inhalte konzentrieren. Trainingsinhalte können kulturspezifisch oder kulturallgemein sein. Trainingsmethoden können informationsorientiert oder erfahrungsorientiert sein.

Nach Gudykunst und Hammer (1983) können aus der Kombination von Inhalten und Methoden vier unterschiedliche Trainingskonzepte entstehen, was sich auch mit den Ergebnissen von Zhang und Zhou (2019) deckt. Diese werden im Folgenden kurz erläutert:

1. kulturallgemein-informationsorientiert - bieten einen hohen kognitiven Lerneffekt in Bezug auf das Verständnis interkultureller Kommunikationsprozesse.

2. kulturspezifisch informationsorientiert - umfassen Informationen zum Zielland und zu verschiedenen Kulturen.

3. kulturallgemein-erfahrungsorientiert - allgemein kultursensibilisierende Simulationen und Rollenspiele, wobei affektive und verhaltensorientierte Lernziele im Vordergrund stehen.

4. kulturspezifisch-erfahrungsorientiert - ,Sensitivity Trainings“, Planspiele und andere Simulationsverfahren, die als Trainings off-the-job bi-kulturelle Teilnehmergruppen voraussetzen; Ziel ist eine Auseinandersetzung mit Vorurteilen, Stereotypen und Verhaltenskonventionen; dienen vor allem zur Vorbereitung auf Auslandsaufenthalte, gängige Methode ist der „Cultural Assimilator“.

Das interkulturelle Training dient dazu, bei den Teilnehmenden Wissen über verschiedene Kulturen aufzubauen, welches dazu beiträgt Unterschiede und Gemeinsamkeiten zwischen Kulturen zu erkennen und das eigene Handeln zu reflektieren. Darauf aufbauend kann sich die Person in interkulturellen Situationen kultursensibel verhalten. Ziel des interkulturellen Lernens ist, dass es durch Horizonterweiterung und Anpassung des Selbstkonzeptes zu einer Änderung der Persönlichkeit kommt (Genkova 2019). Bei der reinen Wissensvermittlung bleibt die Interkulturalität jedoch eher modular und abstrakt für die Teilnehmer (Bolten 2001). Idealerweise sollten daher integrierte Trainings in Bezug auf die Zusammensetzung sowie die Verknüpfung von interaktionsorientierten und informatorischen Aspekten stattfinden, um einen guten Transfer des Gelernten in die Praxis zu ermöglichen (Genkova 
2019). Da in der Lern- und Arbeitsumgebung ein jeweils anderer Kontext vorliegt, ist die Übertragung des Gelernten in die Praxis jedoch oftmals schwierig. Diese hängt einerseits davon ab, wie der Teilnehmer die Ähnlichkeit dieser beiden Umgebungen subjektiv wahrnimmt. Ein weiterer Erfolgsfaktor ist die zeitliche Nähe von Weiterbildung und Arbeitsprozess. Bei einem langen Zeitraum zwischen Weiterbildungsmaßnahme und Anwendung hat der Teilnehmende das Gelernte bereits wieder vergessen, stellt Bergmann (2003), basierend auf eigener und fremder Erfahrung, in einem Kapitel eines Handbuches zur Trainingsgestaltung fest. Bergmann (2003) nennt zudem ,,begleitetes Projektlernen [als] eines der zentralen Qualitätsmerkmale“. Durch dieses wird die Differenz von Lernfeld und ,normaler“ Tätigkeit reduziert und ein höherer Grad der Zielerreichung sowie Lerntransfer erlangt.

\subsection{Evaluation interkultureller Trainings und Coachings}

Um Weiterbildungskonzepte anhand des Grades, wie Ziele erreicht werden, zu bewerten, muss zunächst geklärt werden, was den Erfolg einer Weiterbildungsmaßnahme ausmacht. Entscheidend hierbei ist, wie viel des Erlernten die Teilnehmenden im Anschluss an das Training in die Praxis umsetzen. Wie bereits erläutert, sind Trainings eher wissensorientiert und weniger persönlich als Coachingprozesse. Der Unterschied zwischen Trainings- und Arbeitskontext ist zumeist höher als beim Coaching. Daher ist der Transfererfolg häufig geringer ausgeprägt (Barmeyer 2002). Zhang (2016) fand anhand einer kleineren quantitativen Untersuchung mit 85 chinesischen Studierenden im Rahmen einer Dissertation heraus, dass interkultureller Kompetenzgewinn über einen gewissen Zeitraum vom Alter der Personen abhängt. Jüngere Studierende zeigten demnach einen größeren Kompetenzgewinn als ältere. Dabei beschrieb er diesen Zusammenhang jedoch rein deskriptiv, sodass keine Schlüsse auf unterschiedliches Lernverhalten gezogen werden können.

Laut Genkova (2019) ist die Voraussetzung für interkulturelles Lernen eine gewisse Bereitschaft dazu. Kauffeld (2016) beschreibt, dass die Bereitschaft zur Teilnahme an Personalentwicklungsmaßnahmen mit dem Alter abnimmt. Bezüglich interkulturellen Trainings wurden analoge Zusammenhänge noch nicht überprüft, es wird aber angenommen, dass ältere Teilnehmerinnen und Teilnehmer weniger an interkulturellen Trainings teilnehmen und seltener ins Ausland gehen.

Neben dieser Hypothese prüft die vorliegende Studie, ob Personen mit einem absolvierten interkulturellen Training interkulturell kompetenter auf den beschriebenen Lernzielebenen sind und über mehr Offenheit, Einfühlungsvermögen und Flexibilität (in Anlehnung an Van der Zee und Van Oudenhoven 2000) verfügen, als solche, die kein Training absolviert haben. Darüber hinaus wird die Ausländerfeindlichkeit als abhängige Variable betrachtet.

Wie bereits beschrieben, konnten verschiedene Studien (Crisp und Turner 2012; Meleady und Seger 2017) zeigen, dass durch intensive, nicht erfahrungsorientierte Auseinandersetzung mit der jeweiligen Outgroup negative Einstellungen reduziert werden konnten. Genkova (2016) beschreibt darüber hinaus, dass interkulturelle Kompetenz mit einer geringeren Xenophobie einhergeht. Im Zusammenhang mit Personen, die keine Auslandserfahrung haben, ist die Ausländerfeindlichkeit darüber hinaus von besonderer Bedeutung als affektiver Aspekt der interkulturellen 
Kompetenz im Umgang mit Immigranten. Eine positive Einstellung zu kultureller Vielfalt ist Prädiktor für eine erfolgreiche, wertschätzende Zusammenarbeit (Van Dick und Stegmann 2016). Um die Einstellungsebene, die als guter Prädiktor für interkulturelles Verhalten gesehen wird (z. B. Uslucan und Brinkmann 2013; Van Dick und Stegmann 2016), zu untersuchen, wird daher überprüft ob Teilnehmerinnen und Teilnehmer nach einem interkulturellen Training weniger ausländerfeindliche Einstellungen haben als solche ohne Training.

Die Evaluierung verhaltens- und prozessbezogener Maßnahmen stellt sich im Gegensatz zur Evaluierung fachlicher Bildungsmaßnahmen oft als sehr schwierig dar (Götz 2000). Häufig angewendet wird das Evaluationsmodell nach Kirkpatrick (1975). Dieses Stufenmodell teilt die Erfolgsmessung in die vier Stufen Zufriedenheit mit der Weiterbildungsmaßnahme, Lernerfolg (Wissenserweiterung), Transfererfolg (Umsetzung in die Praxis) und Unternehmenserfolg (Kirkpatrick und Kirkpatrick 2006) ein.

Da eine ausführliche Messung der Transferleistung über einen längeren Zeitraum den Rahmen vieler Studien sprengt, diskutierten Orazbayeva et al. (2016) für den Transfer die allgemeine interkulturelle Kompetenz $\mathrm{zu}$ erfassen und sprechen sich trotz methodischer Einschränkungen für diese Herangehensweise aus. Einschränkungen ergeben sich durch die veränderte Perspektive (ausschließlich individuell im Vergleich zu individuell, sozial, organisational) und die kurzfristige Betrachtungsweise. Demgegenüber steht die Betrachtung einzelner Zusammenhänge, die ein Inkrement zu einer Theorie leisten können, in diesem Fall zu Zusammenhängen zwischen interkulturellen Trainings und interkultureller Kompetenz.

\section{Fragestellung, Methodik und Stichprobenbeschreibung}

\subsection{Fragestellung}

Es wurden im Rahmen der Studie folgende Hypothesen aufgestellt:

Hypothese 1 Zusammenhang von Alter und Häufigkeit von interkulturellen Trainings sowie Auslandsaufenthalten.

- 1a. Jüngere Personen nehmen häufiger an interkulturellen Trainings teil als ältere Personen.

- 1b. Jüngere Personen gehen häufiger für längere Zeit ins Ausland als ältere Personen.

Hypothese 2 Zusammenhang interkultureller Trainings mit den drei Lernzielebenen interkultureller Kompetenz.

- 2a. Kognitive Komponente: Teilnehmer interkultureller Trainings setzen sich auf kognitiver Ebene bewusster mit ihrer eigenen Identität auseinander als „Untrainierte“. 
- 2b. Affektive Komponente: Teilnehmer interkultureller Trainings haben in affektiver Hinsicht eine positivere Einstellung bezüglich ihrer eigenen Identität als „Untrainierte“.

- 2c. Verhaltensbezogene Komponente: Teilnehmer interkultureller Trainings verhalten sich Menschen mit anderem kulturellem Hintergrund gegenüber effizienter und angemessener als ,Untrainierte“.

Hypothese 3 Zusammenhang von interkulturellen Trainings mit den für interkulturelle Kompetenz wichtigen Eigenschaften „Offenheit“, „Einfühlungsvermögen“, „Flexibilität“, diese sind ausgeprägter bei den Teilnehmenden von interkulturellen Trainings als bei den „Untrainierten“.

Hypothese 4 Zusammenhang von Ausländerfeindlichkeit und interkulturellem Training: Menschen, die noch an keinem interkulturellen Training teilgenommen haben, haben ausländerfeindlichere Neigungen als solche, die an interkulturellen Trainings teilgenommen haben.

\subsection{Methodik}

Um die Hypothesen zu prüfen, wurde eine korrelative Querschnittsstudie durchgeführt. Die Durchführung erfolgte von Juni bis August 2019 mittels Paper-Pencil Fragebögen. Die Teilnehmerinnen und Teilnehmer willigten auf einem separaten Blatt schriftlich ein, dass ihre Daten zu rein wissenschaftlichen Zwecken verwendet werden dürften. Darauf folgte eine Instruktion der Befragten auf die folgenden Fragen möglichst spontan und ehrlich zu antworten. Im ersten Abschnitt wurden demographische Daten, die Teilnahme an interkulturellen Trainings (Ja/Nein) sowie die Anzahl der Auslandsaufenthalte abgefragt.

Der zweite Abschnitt maß die Ausprägung der kognitiven, affektiven und verhaltensbezogenen Ebenen mithilfe des ,Multigroup Ethnic Identity Measure“ (MEIM) nach Phinney et al. (1999). In der MEIM weist eine mittlere interne Konsistenz $\alpha=0,72$ auf und enthält 23 Items, welche auf einer fünfstufigen Likert-Skala basieren (Phinney et al. 1999).

Der nächste Abschnitt erfasste die Dimensionen „kulturelle Empathie“, „Offenheit“, ,,soziale Initiative“, ,,emotionale Stabilität“ und „Flexibilität“ mit der ,Multicultural Personality Questionnaire“ (MPQ), welche im Jahr 2000 von Van der Zee und Van Oudenhoven entwickelt wurde. Diese dient der Messung der interkulturellen Kompetenz der Probanden. Die MPQ enthält 91 Items, welche ebenfalls auf einer fünfstufigen Likert-Skala basieren. Sie weist eine hohe Reliabilität von $\alpha=0,87$ auf.

Der letzte Abschnitt erfasste das Ausmaß der Ausländerfeindlichkeit der Befragten. Hierfür wurde eine gekürzte Fassung der Skala aus dem Erhebungsinstrument zur Schülerbefragung des Kriminologischen Forschungsinstituts Niedersachsen von Wetzels, Gabriel und Pfeiffer verwendet. Die ursprüngliche Untersuchung aus dem Jahr 2000 hatte zum Ziel, die Integrationschancen und -probleme junger Aussiedler zu analysieren (Strobl und Kühnel 2000). Insgesamt wurde die Ausländerfeindlich- 
keit über acht Fragen anhand einer vierstufigen Likert-Skala erhoben. Die interne Konsistenz fiel mit $\alpha=0,50$ fragwürdig bis gerade noch akzeptabel aus.

Analog zur Untersuchung von Zhang wurden die Befragten in Jüngere (jünger als 35 Jahre) und Ältere eingeteilt. Ein Auslandsaufenthalt wurde in Anlehnung an Genkova (2019) als ein Aufenthalt im Ausland von mehr als drei Monaten operationalisiert, da ab dieser Zeit relevante Effekte der Akkulturation auftreten.

\subsection{Stichprobenbeschreibung}

Bei der Akquise war es besonders wichtig, eine möglichst gleichmäßige Anzahl an Personen, die an interkulturellen Trainings teilgenommen haben und solchen, die noch nie ein interkulturelles Training besucht hatten, zu erreichen. Dementsprechend wurde ein Oversampling vorgenommen. Es wurden die Teilnehmenden eines Seminars „Grundlagen der Interkulturellen Kommunikation“ an der Universität Passau gewählt, das sowohl kulturspezifisch, als auch kulturell allgemeine Inhalte aufgreift und dadurch sowohl sensibilisiert als auch Grundlagenwissen zu verschiedenen Kulturräumen schafft. Interaktionsorientierte Inhalte sind nicht vorgesehen. Die Befragten wurden jeweils unmittelbar nach Absolvieren des letzten Trainingsteils gebeten an der Umfrage teilzunehmen da sie alle an dem gleichen Training in geschütztem Umfeld teilgenommen hatten.

Die Vergleichsgruppe, welche aus interkulturell untrainierten Personen bestand (,Untrainierte“), wurde aus Studierenden anderer Fachrichtungen sowie dem Freundes- und Bekanntenkreis gebildet. So konnte ein Querschnitt aus verschiedensten Alters-, Bildungs- und Berufsgruppen erreicht werden. In dieser Befragung hatten $40 \%$ an einem oder (in der Vergangenheit dann) an mehreren interkulturellen Trainings teilgenommen. Demgegenüber standen $60 \%$ „Untrainierte“. Von insgesamt etwa 130 verbreiteten Fragebögen konnten 101 in die Auswertung aufgenommen werden. Die Rücklaufquote betrug somit $78 \%$.

$57 \%$ der Probanden waren weiblichen, $44 \%$ männlichen Geschlechts, niemand hatte divers angegeben. Die Befragten waren zwischen 14 und 69 Jahre alt, wobei das Durchschnittsalter 27,68 Jahre betrug. 89 der 101 Probanden wurden in Deutschland geboren, 12 dagegen im Ausland. 95\% waren deutscher, 5\% anderer Nationalität. $60 \%$ der Probanden hatten das Abitur als höchsten Bildungsabschluss, $22 \%$ einen mittleren Bildungsabschluss, $8 \%$ ein abgeschlossenes Studium, $7 \%$ einen Hauptschulabschluss. $2 \%$ der Probanden hatten promoviert. 59\% hatten noch nie längere Zeit im Ausland verbracht, $41 \%$ gaben an, schon mindestens einmal für mindestens drei Monate am Stück im Ausland gewesen zu sein. Die Länge der Aufenthalte reichte dabei von 3 Monaten bis hin zu 21 Jahren, wobei die Länge 6 Monate die höchste Häufigkeit aufwies. Generell konnten sich $88 \%$ gut vorstellen, einmal selbst im Ausland zu leben, nur $11 \%$ lehnten dies grundsätzlich ab.

\section{Ergebnisse}

Die Normalverteilung der untersuchten Merkmale wurde mit dem KomolgorovSmirnov Test bestätigt. 


\subsection{Zusammenhang Alter - interkulturelles Training - Auslandsaufenthalt}

Zunächst wird untersucht, ob zwischen dem Alter und der Teilnahme an interkulturellen Trainings (H1a) sowie zwischen dem Alter und der Tendenz, längere Zeit im Ausland zu verbringen (H1b), ein Zusammenhang besteht.

Die Kreuztabelle für H1a zeigt, dass 38 Personen $(45,8 \%$ ) der ersten Altersgruppe und zwei Personen $(11,1 \%)$ der zweiten Altersgruppe an interkulturellen Trainings teilgenommen haben. Die prozentualen Verteilungen unterscheiden sich also erheblich voneinander. Daher kann auf einen statistischen Zusammenhang zwischen den Merkmalen Altersgruppe und interkulturelles Training geschlossen werden. Durch den Chi-Quadrat-Test wird nun überprüft, ob der Unterschied zwischen den Altersgruppen signifikant ist. Der Chi-Quadrat-Test zeigt ein hoch signifikantes Ergebnis $x^{2}(1)=7,43 ; p<0,01$. Es nehmen also signifikant mehr junge Leute an interkulturellen Trainings teil als ältere. H1a kann somit bestätigt werden.

H1b überprüft im Anschluss den Zusammenhang zwischen Alter und Auslandsaufenthalt. In der jüngeren Altersgruppe haben mit 88,6\% deutlich mehr Befragte eine längere Zeit im Ausland verbracht, als in der älteren Vergleichsgruppe mit 12,5\%. Die Signifikanz dieses Unterschieds wird im Anschluss erneut mit dem ChiQuadrat-Test überprüft. Der Chi-Quadrat-Test zeigt ein hoch signifikantes Ergebnis $x^{2}(1)=7,90 ; p<0,01$, womit $\mathrm{H} 1 \mathrm{~b}$ bestätigt wird. In der jüngeren Altersgruppe haben mehr Personen bereits eine längere Zeit im Ausland verbracht.

\subsection{Effekt interkultureller Trainings auf die drei Lernzielebenen interkultureller Kompetenz}

Im Folgenden wird der Zusammenhang zwischen interkulturellen Trainings und den kognitiven, affektiven und verhaltensbezogenen Komponenten interkultureller Kompetenz untersucht. Hierfür werden über t-Tests drei Mittelwertvergleiche durchgeführt.

Zunächst wird H2a überprüft, welcher die Annahme zugrunde liegt, dass Personen, die bereits ein Training erhalten haben sich auf kognitiver Ebene bewusster mit anderen Kulturen auseinandersetzen:

Der t-Test (siehe Tab. 1) zeigt keinen signifikanten Unterschied in Bezug auf die kognitive Ebene $(t(98)=1,22 ; p=0,27 ; r=0,18)$ bei Personen mit interkulturellem Training $(M=3,27 ; S D=0,65)$ und solchen, die kein Training absolviert haben $(M=3,09 ; S D=0,75)$.

Anschließend wird geprüft, ob auf affektiver Ebene ein Unterschied zwischen Trainierten und Untrainierten vorliegt $(\mathrm{H} 2 \mathrm{~b})$.

Wie Tab. 2 zeigt, ergibt der t-Test kein signifikantes Ergebnis $(t(98)=1,87$; $p=0,06 ; r=0,27)$. Es besteht kein signifikanter Unterschied auf affektiver Ebene zwischen trainierten $(M=3,5 ; S D=0,61)$ und untrainierten Befragten $(M=3,08$; $S D=0,76$ ). Daher kann $\mathrm{H} 2 \mathrm{~b}$ nicht bestätigt werden.

Hypothese H2c untersucht schließlich, ob interkulturell Trainierte auf verhaltensbezogener Ebene stärkere Ausprägungen aufweisen als Untrainierte (Tab. 3).

Der t-Test zeigt, dass ein signifikanter Mittelwertunterschied vorliegt $(t(98)=2,45$; $p<0,05)$. Interkulturell trainierte Teilnehmerinnen und Teilnehmern $(M=4,06$; 


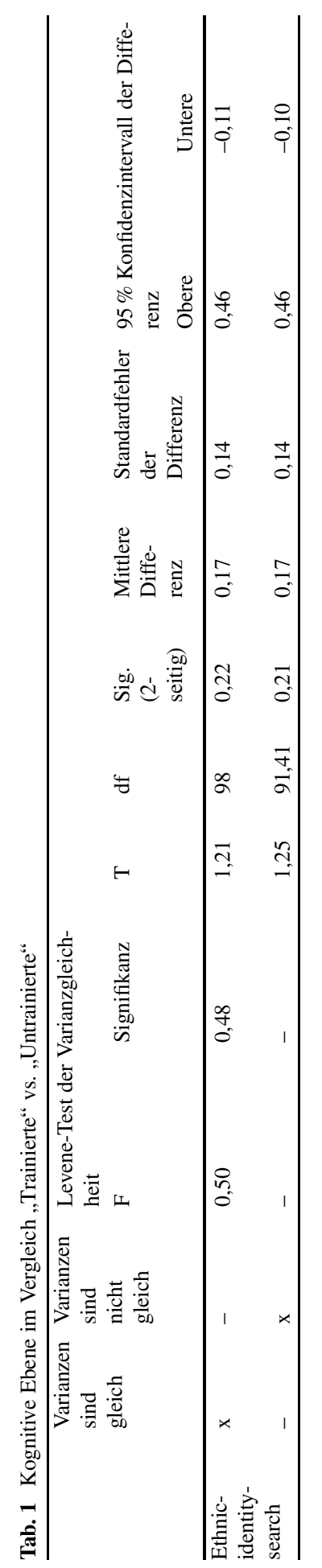




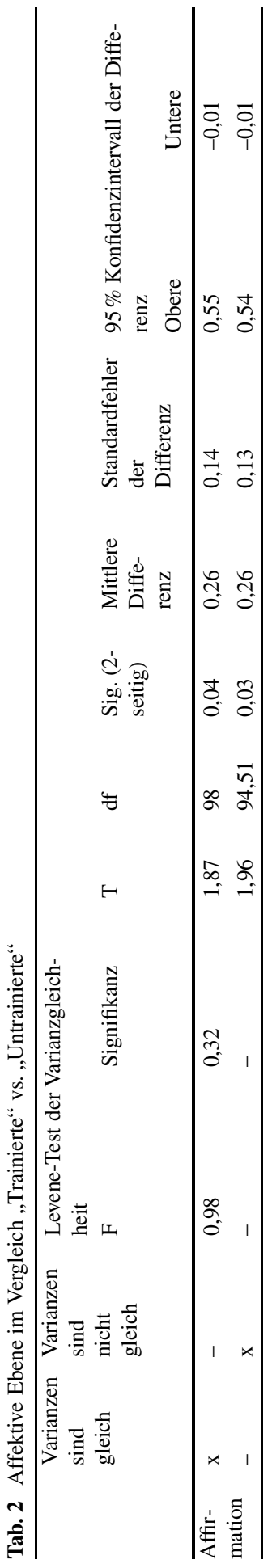




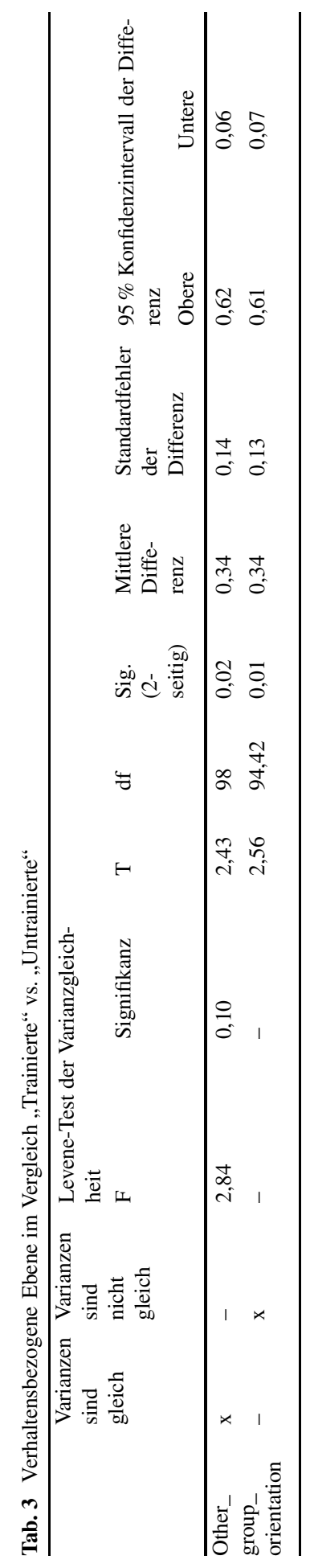


$S D=0,60)$ verhalten sich interkulturell kompetenter als die Untrainierten $(M=3,71$; $S D=0,74)$. Trainierte treten aktiver mit Personen aus anderen Volksgruppen in Beziehung und tauschen sich mit diesen aus. Zudem verbringen diese Personen im Schnitt mehr Zeit mit kulturfremden Personen als solche, die nie an Trainings teilgenommen haben. $\mathrm{H} 2 \mathrm{c}$ ist somit bestätigt.

\subsection{Effekt interkultureller Trainings auf die interkulturelle Kompetenz}

Wie erwähnt, sind insbesondere drei Eigenschaften in Bezug auf die interkulturelle Kompetenz einer Person von Bedeutung: Offenheit, Einfühlungsvermögen und Flexibilität. Die dritte Hypothese untersucht, inwieweit die relevanten Eigenschaften sich bei Menschen, die an Trainings teilgenommen haben, von jenen unterscheiden, die nie Teilnehmer eines interkulturellen Trainings waren. Dies wurde mittels t-Tests überprüft (siehe Tab. 4):

Für die Variable Offenheit zeigt der t-Test einen höchst signifikanten Mittelwertunterschied $(t(98)=4,42 ; p<0,01 ; r=0,37)$. Interkulturell Trainierte $(M=3,73$; $S D=0,36)$ haben eine höhere Offenheit als untrainierte Personen $(M=3,36$; $S D=0,45$ ), eine unvoreingenommenere Wahrnehmung der kulturellen Unterschiede und Ähnlichkeiten und eine tolerantere Haltung gegenüber Neuem.

Der Mittelwertvergleich für die Variable Einfühlungsvermögen ist nicht signifikant $(t(98)=0,96 ; p=0,34 ; r=0,09)$.

Für die dritte Eigenschaft Flexibilität zeigt sich ein signifikanter Unterschied $(t(98)=2,30 ; p<0,05 ; \quad r=0,19)$ zwischen Personen mit Training $(M=3,23$; $S D=0,37)$ und Personen ohne Training $(M=3,04 ; S D=0,42)$. Es besteht demnach ein Zusammenhang zwischen Flexibilität, sein eigenes Verhalten anzupassen und entsprechend auf neue und fremdartige Situationen zu reagieren und interkulturellen Trainings.

\subsection{Zusammenhang Ausländerfeindlichkeit - interkulturelles Training}

Schließlich wird der Zusammenhang zwischen Ausländerfeindlichkeit und interkulturellen Trainings betrachtet. Der t-Test, zeigt ein signifikantes Ergebnis $(t(98)=3,03 ; p<0,01 ; r=0,29$; Tab. 5). Teilnehmer von interkulturellen Trainings $(M=1,45, S D=0,44)$ weisen eine geringere Neigung zu Ausländerfeindlichkeit auf als interkulturell ,Untrainierte“ $(M=1,74, S D=0,51)$. Hypothese 4 wird somit bestätigt.

\section{Diskussion}

Die zentrale Fragestellung dieser Studie war, welche Zusammenhänge zwischen Trainings, die ohne prozessbegleitendes Coaching stattfinden, und interkultureller Kompetenz bestehen.

Es wurde gezeigt, dass jüngere Personen öfter an interkulturellen Trainings teilnehmen und mehr Zeit im Ausland verbringen. Dies kann womöglich dadurch begründet werden, dass jüngere Menschen bereits in einer zunehmend globalisierten 


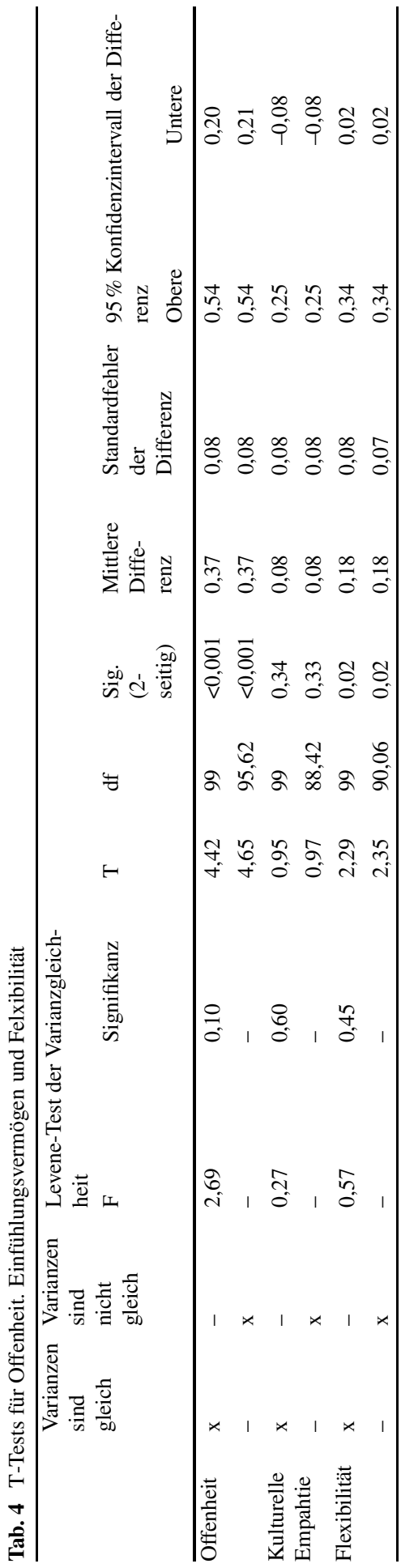




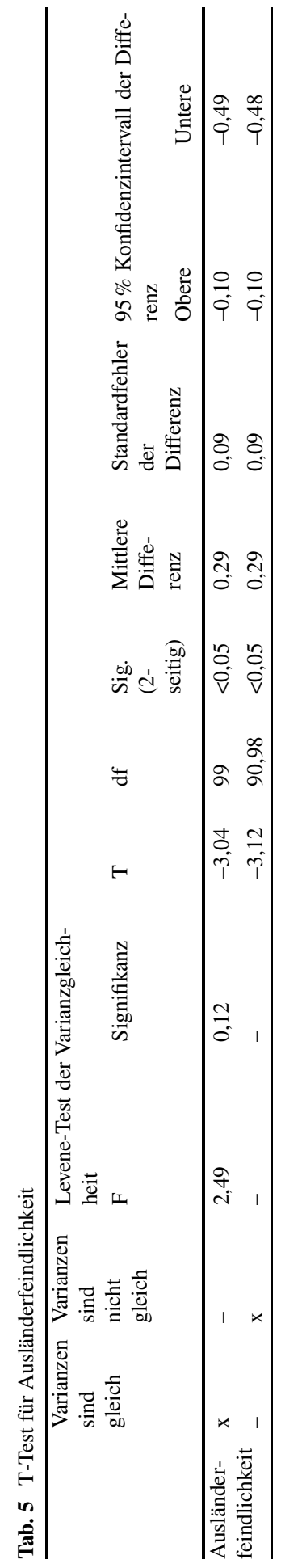


Gesellschaft aufgewachsen sind und in jungen Jahren in Kontakt mit anderen Kulturen gekommen sind. Analog attestiert Bittner (2003) der jüngeren Generation der westlichen Welt eine vergleichsweise hohe Offenheit im Vergleich zu älteren Generationen, trotz eines gewissen Ethnozentrismus. So kann es sein, dass jüngere Personen den Bedarf an interkultureller Handlungskompetenz in einem stärkeren Maße anerkennen. Es sind jedoch auch andere Einflussgrößen denkbar.

Die Möglichkeiten für international Studierende wurden in den letzten 20 Jahren extrem ausgebaut, was zu einem Anstieg der international Studierenden von 2 im Jahr 2000 auf 5,3 Mio. im Jahr 2017 geführt hat (UNESCO 2020). Interkulturelle Trainings als Weiterbildungsmaßnahme gewinnen insgesamt an Bedeutung (Genkova 2019). Es ist daher anzunehmen, dass die Ergebnisse dieser Studie lediglich eine Momentaufnahme darstellen und sich das Bewusstsein für interkulturelle Kompetenz übergreifend ausbreitet (vgl. Matveev 2017). Ebenso verhält es sich bei Auslandsaufenthalten, die im Berufsleben inzwischen gewünscht und gefordert werden, noch vor 20 Jahren aber eher eine Besonderheit waren (Barmeyer 2002). Eine Folgestudie sollte daher neben dem Alter und der Teilnahme an einem Training oder Auslandsaufenthalt auch die Motivation dazu mit in Betracht ziehen, wie es Holtbrügge und Engelhard (2016) für Auslandsaufenthalte in einer Querschnittsstudie $(N=901)$ taten. Neben Erkenntnissen über die Wirkungen von Intergruppenkontakt konnten sie zeigen, dass die Motivation etwas über eine Kultur zu lernen oder den eigenen Horizont zu erweitern, sowie die Motivation eine bestimmte Universität zu besuchen positiv mit der interkulturellen Kompetenz zusammenhingen, während die Motivation eine gute Zeit zu haben negativ mit der Kompetenz zusammenhing. Karriereorientierte Motivation hatte keinen Zusammenhang mit der Kompetenz. Zukünftige Studien sollten diesen Faktor in ihre Überlegungen miteinbeziehen.

Zudem wurden die Zusammenhänge zwischen interkulturellen Trainings und den für interkulturelle Handlungskompetenz wichtigen kognitiven, affektiven und verhaltensbezogenen Faktoren untersucht. Die Analyse ergab keine signifikanten Unterschiede auf kognitiver oder affektiver Ebene. Für die verhaltensbezogene Ebene wurde jedoch ein signifikanter Unterschied gezeigt. Dabei muss berücksichtigt werden, dass es sich bei der erhobenen Studie um eine Selbstbeurteilung handelt, die lediglich die subjektive Meinung der Befragten widerspiegelt. Dennoch stellt sie einen annehmbaren Prädikator für späteres Verhalten dar (Orazbayeva et al. 2016). Diese Ergebnisse lassen darauf schließen, dass einmalige ein- bis zweitägige Trainings alleine nicht mit einer langfristigen Wissens- und Einstellungsänderung zusammenhängen. Es bedarf weiterer Maßnahmen, um die Inhalte der Trainings nachhaltig bei den Teilnehmern zu festigen. Zwar ist ein erster Erfolg der Trainings in Bezug auf interkultureller Handlungskompetenz bei der verhaltensbezogenen Komponente anzunehmen, dieser könnte jedoch in Kombination mit signifikanten nachhaltigen Verbesserungen auf kognitiver und affektiver Ebene deutlich erhöht werden.

Kraut (2003) führt an, dass die Änderung von Einstellungen ein deutlich komplexerer und langfristigerer Prozess ist als die Anpassung von Verhalten. Es konnte dennoch eine niedrigere Ausländerfeindlichkeit bei Trainierten im Vergleich zu den untrainierten Teilnehmenden gefunden werden. Dies steht im Konflikt damit, dass auf affektiver und kognitiver Ebene keine signifikanten Unterschiede gefunden werden konnten. Betrachtet man die modernen Theorien zur Bildung von Vorurteilen 
und negativen Einstellungen gegenüber der Outgroup, wie Pettigrew (2016) sie in einer Zusammenfassung seiner Forschungsergebnisse elaboriert, sollte man annehmen, dass die Eigenschaften unvoreingenommen und positiv Menschen aus anderen Kulturen gegenüber zu sein und sich aktiv mit der eigenen und fremden kulturellen Prägung auseinanderzusetzen stark mit der Ausprägung negativer Einstellungen zu Immigranten zusammenhängt. Sowohl die affektive als auch die kognitive Dimension basieren auf der sozialen Kategorisierung, der Salienz einer bestimmten Kategorie und der Verfügbarkeit von Ressourcen kulturell verankerte negative Prädispositionen aktiv zu verarbeiten (Pettigrew 2016). Intergruppenkontakt als wichtiges Konzept zur Reduktion negativer Einstellungen (Pettigrew 2016) kommt bei einem rein informationsorientierten Training als Erklärung logischerweise nicht in Frage. Hier sollte in Betracht gezogen werden, dass ein Training möglicherweise nicht zwangsweise mit einer Einstellungsänderung zusammenhängt, sondern mit einer Sensibilisierung für politisch korrekte Äußerungen und Verhaltensweisen.

Aus einer wirtschaftspsychologischen Perspektive wird zwar stets ein Bezug zum tatsächlichen Verhalten im Sinne der prädiktiven Validität gesucht (Kanning 2019). Dennoch zeigen Studien zu Einstellungen zu kultureller Vielfalt (z. B. Kauff et al. 2019; Gutentag et al. 2018), dass langfristige Änderung von kulturellen Einstellungen sowohl in Unternehmen, als auch in der Lehre zu nachhaltig besseren Ergebnissen führen. Dies kann beispielsweise durch auf das Training folgende prozesshafte Maßnahmen wie Coachings erreicht werden. Um nachhaltige, sprich langfristige Effekte zu untersuchen, sollten zukünftige Studien Längsschnitt-Designs in Betracht ziehen. Dies würde es darüber hinaus ermöglichen kausale Zusammenhänge zu beobachten.

Die Untersuchung der Charaktereigenschaften „Offenheit“, „Einfühlungsvermögen“ und „Flexibilität“, welche für interkulturelle Handlungskompetenz von großer Bedeutung sind, zeigt bei zwei der Eigenschaften Unterschiede. So zeigten Trainingsteilnehmerinnen und Teilnehmer eine offenere Haltung gegenüber Fremdkulturellem und flexiblere Reaktionen auf Neues als diejenigen, die kein Training absolviert hatten. Für das Konstrukt Offenheit sind die gleichen Bedenken bezüglich sozial erwünschten Antwortverhaltens relevant wie für die Ausländerfeindlichkeit. Auf der anderen Seite zeigten Meleady und Seger (2017) in einem experimentellen Studiendesign, dass die Auseinandersetzung mit der Outgroup auch kurzfristig dazu beiträgt die Offenheit gegenüber dieser Gruppe zu erhöhen und negative Einstellungen abzubauen, sowie Vertrauen zu bilden. Um einen solchen Effekt auszuschließen, wären implizite Assoziationstests eine mögliche Option für zukünftige Studien.

Für die vorliegende Studie gelten einige weitere Limitationen. Aus dem querschnittlichen Design ergibt sich, dass Aussagen über kausale Zusammenhänge nicht getroffen werden können. Darüber hinaus ist die Stichprobe vergleichsweise klein. Es kann nicht davon ausgegangen werden, dass sich die Ergebnisse reibungslos auf einen Arbeitskontext übertragen lassen. Es sollte angemerkt werden, dass unter der Prämisse, dass eine Messung der Kompetenz eine angemessene Operationalisierung des Trainingserfolges darstellt, die grundlegenden Zusammenhänge vergleichbar sind. Es ist jedoch davon auszugehen, dass Kontextfaktoren, wie eine Familie, die Auslandsaufenthalte verhindert, zu unterschiedlichen Ergebnissen führen würden. Zukünftige Studien sollten daher ein vergleichendes Evaluationsdesign 
in Betracht ziehen und Teilnehmende eines wirtschaftlich orientierten Trainings mit und ohne Coaching vergleichen, sowie eine Kontrollgruppe. Zuletzt ist der Aspekt der sozialen Erwünschtheit zu diskutieren. Für das Konstrukt Ausländerfeindlichkeit ist anzunehmen, dass eine verstärkte Tendenz zu sozial erwünschten, politisch korrekten Antworten vorlag. Diese könnte auch die geringe interne Konsistenz der Messung erklären. Eine differenziertere Betrachtung des Konstruktes würde sowohl dieses Problem lösen als auch detailliertere Aussagen über Zusammenhänge mit interkulturellen Trainings ermöglichen. Für zukünftige Studien wird daher vorgeschlagen eine detailliertere Betrachtung der Ausländerfeindlichkeit und möglicherweise unterschiedlicher Trainingstypen vorzunehmen. Dies wäre besonders vor dem Hintergrund von Diversitätseinstellungen als Prädiktor für Team-Performance (Van Dick und Stegmann 2016) relevant.

Obwohl diese Studie in einem universitären Kontext durchgeführt wurde, weist das untersuchte Training viele Äquivalenzen mit Trainings im wirtschaftlichen Kontext auf. So fand es vom restlichen Lehrplan unabhängig, sozusagen off-the-job statt, war eine geschlossene Einheit, und befasste sich mit allgemeinen und kulturspezifischen, informationsorientierten Inhalten, was laut Bittner (2003) der üblichen wirtschaftlichen Praxis entspricht. Es wird daher angenommen, dass die Ergebnisse auf einen wirtschaftlichen Kontext übertragbar sind, mit Ausnahme der Aussagen zu den Altersgruppen, da hier andere Kontextfaktoren zu erwarten sind.

Zusammenfassend kann gesagt werden, dass interkulturelle Trainings mit Unterschieden in der interkulturellen Kompetenz der Teilnehmenden zusammenhängen. Es konnte gezeigt werden, dass diejenigen Teilnehmenden, die ein informationsorientiertes interkulturelles Training absolviert hatten, eine höhere Offenheit und Flexibilität, sowie bessere Werte in der verhaltensbezogenen Lernzielebene und niedrigere Ausprägungen von Ausländerfeindlichkeit zeigten. Jedoch können durch ein Training nicht alle relevanten Eigenschaften und Bereiche für interkulturelle Handlungskompetenz gefördert werden. Die Untersuchten zeigten keine Unterschiede hinsichtlich der kulturellen Empathie, sowie auf der affektiven und kognitiven Lernzielebene. Es ist anzunehmen, dass es dazu langfristiger Begleitmaßnahmen bedarf. Ein Training ist vor allem als Vorbereitung und Ausgangspunkt für diese geeignet. Erste positive Ergebnisse müssen dann im Weiteren aufgegriffen, ausgebaut und in die Praxis umgesetzt sowie in ständiger Reflexion einer Prüfung unterzogen werden. Interkulturelle Trainings ohne prozessbegleitende Coachings sind also zu einem gewissen Grad sinnvoll, aber nicht darüber hinaus. Die Sinnhaftigkeit und der Effekt der Maßnahme kann allerdings in Kombination mit längerfristig angelegten Weiterbildungsmaßnahmen deutlich verbessert werden. Daher sollten Unternehmen zukünftige integrierte Lernkonzepte einsetzen, um ihre Mitarbeiter optimal auf eine interkulturelle Arbeitssituation vorzubereiten. Für den Bildungskontext gelten diese Aussagen analog. Hier stellen Mentoring Programme (Begleitung des Mentees durch ein erfahrenes Peer Mitglied) ein probates Mittel für begleitende Maßnahmen von einzelnen Trainings oder Programmen dar. Entscheidender als die Ausgestaltung einer begleitenden Maßnahme ist laut Bittner (2008), dass prozessbegleitende Reflexionsphasen unter Anleitung bereits bei der Konzeption eines Trainings berücksichtigt werden. 
Open Access Dieser Artikel wird unter der Creative Commons Namensnennung 4.0 International Lizenz veröffentlicht, welche die Nutzung, Vervielfältigung, Bearbeitung, Verbreitung und Wiedergabe in jeglichem Medium und Format erlaubt, sofern Sie den/die ursprünglichen Autor(en) und die Quelle ordnungsgemäß nennen, einen Link zur Creative Commons Lizenz beifügen und angeben, ob Änderungen vorgenommen wurden.

Die in diesem Artikel enthaltenen Bilder und sonstiges Drittmaterial unterliegen ebenfalls der genannten Creative Commons Lizenz, sofern sich aus der Abbildungslegende nichts anderes ergibt. Sofern das betreffende Material nicht unter der genannten Creative Commons Lizenz steht und die betreffende Handlung nicht nach gesetzlichen Vorschriften erlaubt ist, ist für die oben aufgeführten Weiterverwendungen des Materials die Einwilligung des jeweiligen Rechteinhabers einzuholen.

Weitere Details zur Lizenz entnehmen Sie bitte der Lizenzinformation auf http://creativecommons.org/ licenses/by/4.0/deed.de.

\section{Literatur}

Barmeyer, C. (2010). Interkulturalität. In C. Barmeyer, P. Genkova \& J. Scheffer (Hrsg.), Interkulturelle Kommunikation und Kulturwissenschaft: Grundbegriffe, Wissenschaftsdisziplinen, Kulturräume (2. Aufl. S. 129-155). Passau: Stutz.

Barmeyer, C.I. (2002). Interkulturelles Coaching. In C. Rauen (Hrsg.), Handbuch Coaching (2. Aufl. S. 199-231). Göttingen: Hogrefe.

Bergmann, G. (2003). Bildungscontrolling - Transfer - Evaluation. In L. Hofmann (Hrsg.), Innovative Weiterbildungskonzepte. Trends, Inhalte und Methoden der Personalentwicklung in Unternehmen (3. Aufl. S. 267-279). Göttingen: Hogrefe.

Bittner, A. (2003). Interkulturelles Training: Mehr Schein als Sein? In L. M. Hofmann \& E. Regnet (Hrsg.), Innovative Weiterbildungskonzepte. Trends, Inhalte und Methoden der Personalentwicklung in Unternehmen (3. Aufl. S. 113-123). Göttingen: Hogrefe.

Bittner, A. (2008). Damit muss man eben leben - psychologische Barrieren bei interkulturellen Trainings. Wirtschaftspsychologie aktuell, 1, 28-31.

Bolten, J. (2001). Interkulturelles Coaching, Mediation, Training und Consulting als Aufgaben des Personalmanagements internationaler Unternehmen. In A. Clermont (Hrsg.), Strategisches Personalmanagement in globalen Unternehmen (S. 909-926). München: Vahlen.

Bolten, J. (2006). Interkultureller Trainingsbedarf aus der Perspektive der Problemerfahrungen entsandter Führungskräfte. Interkulturelles Lernen/Interkulturelles Training, 6, 57-75.

Crisp, R. J., \& Turner, R. N. (2012). The imagined contact hypothesis. In Advances in experimental social psychology (Bd. 46, S. 125-182). New York, Boston, London, Oxford: Academic Press.

Geeraert, N., \& Demes, K. (2012). The impact of living abroad: research report. University of Essex

Genkova, P. (2016). Experience abroad and its relation to intercultural competence and cross-cultural tolerance. International Journal of Business and Management, 11(5), 1-11.

Genkova, P. (2019). Interkulturelle Wirtschaftspsychologie. Berlin, Heidelberg, New York: Springer.

Götz, K. (2000). Management-Training bei DaimlerChrysler. In M. Welge, K. Häring \& A. Voss (Hrsg.), Management Development (S. 327-345). Stuttgart: Schäffer-Poeschel.

Gudykunst, W. B., \& Hammer, M. R. (1983). Basic training design: approaches to intercultural training. In D. Landis \& R. W. Brislin (Hrsg.), Issues in the theory and design. Handbook of intercultural training, (Bd. 1, S. 118-154). Elmsford: Pergamon.

Gutentag, T., Horenczyk, G., \& Tatar, M. (2018). Teachers' approaches toward cultural diversity predict diversity-related burnout and self-efficacy. Journal of Teacher Education, 69(4), 408-419.

Holtbrügge, D., \& Engelhard, F. (2016). Study abroad programs: Individual motivations, cultural intelligence, and the mediating role of cultural boundary spanning. Academy of Management Learning \& Education, 15(3), 435-455.

Kanning, U.P. (2019). Standards der Personaldiagnostik. Göttingen: Hogrefe.

Kauff, M., Stegmann, S., van Dick, R., Beierlein, C., \& Christ, O. (2019). Measuring beliefs in the instrumentality of ethnic diversity: Development and validation of the Pro-Diversity Beliefs Scale (PDBS). Group Processes \& Intergroup Relations, 22(4), 494-510.

Kauffeld, S. (2016). Nachhaltige Personalentwicklung und Weiterbildung. Berlin, Heidelberg, New York: Springer. 
Kiechl, R. (1997). Interkulturelle Kompetenz. In E. Kopper \& R. Kiechl (Hrsg.), Globalisierung: Von der Vision zur Praxis (S. 11-30).

Kirkpatrick, D. L. (1975). Techniques for evaluating training programs. In D. L. Kirkpatrick (Hrsg.), Evaluating training programs. Alexandria: ASTD.

Kirkpatrick, D.L., \& Kirkpatrick, J.D. (2006). Evaluating training programs. The four levels (3. Aufl.). New York: McGraw-Hill.

Kraut, S. (2003). Interkulturelle Kompetenz durch interkulturelles Training? Eine kritische Analyse ausgewählter Studien zur Effektivität interkultureller Trainings. Unveröffentlichte Diplomarbeit, Universität Passau.

Matveev, A. (2017). Describing intercultural competence. In Intercultural competence in organizations (S. 3-26). Cham: Springer.

Meleady, R., \& Seger, C. R. (2017). Imagined contact encourages prosocial behavior towards outgroup members. Group Processes \& Intergroup Relations, 20(4), 447-464.

Neugebauer, M., Heublein, U., \& Daniel, A. (2019). Studienabbruch in Deutschland: Ausmaß, Ursachen, Folgen, Präventionsmöglichkeiten. Zeitschrift für Erziehungswissenschaft, 22(5), 1025-1046.

Orazbayeva, B., Baaken, T., \& Meerman, A. (2016). Intercultural knowledge transfer in teams. Findings based on a case study. https://www.researchgate.net/publication/305640163_Intercultural_ Knowledge_Transfer_in_Teams_-_Findings_Based_on_a_Case_Study. Zugegriffen: 16. Juni 2020.

Pettigrew, T.F. (2016). In pursuit of three theories: authoritarianism, relative deprivation, and intergroup contact. Annual review of psychology, 67, 1-21.

Phinney, J., Roberts, R., Masse, L., Chen, Y., Roberts, C., \& Romero, A. (1999). The structure of ethnic identity in young adolescents from diverse ethnocultural groups. Journal of Early Adolescence, 19, $301-322$.

Regnet, E., \& Hofmann, L. M. (2003). Mit Weiterbildung die Wettbewerbsfähigkeit steigern. „Lernen ist wie Rudern gegen den Strom, wer aufhört, der treibt zurück“. In L. Hofmann \& E. Regnet (Hrsg.), Innovative Weiterbildungskonzepte. Trends, Inhalte und Methoden der Personalentwicklung in Unternehmen (3. Aufl. S. 13-125). Göttingen: Hogrefe.

Rozkwitalska, M., Chmielecki, M., Przytula, S., Sulkowski, L., \& Basinska, B. A. (2017). Intercultural interactions in multinational subsidiaries. Baltic Journal of Management, 12, 214-239.

Stegmann, S. (2011). Engaging with diversity of social units: A social identity perspective on diversity in organizations (Doctoral dissertation).

Strobl, R., \& Kühnel, W. (2000). Dazugehörig und ausgegrenzt. Analysen zu Integrationschancen junger Aussiedler. Weinheim: Juventa.

Thomas, A., \& Simon, P. (2007). Interkulturelle Kompetenz. In G. Trommsdorff \& H.-J. Konradt (Hrsg.), Anwendungsfelder der kulturvergleichenden Psychologie. Enzyklopädie der Psychologie. Themenbereich C Theorie und Forschung. Serie 7 Kulturvergleichende Psychologie, (Bd. 3, S. 135-177). Göttingen: Hogrefe.

UNESCO (2020). Education: outbound internationally mobile students by host region. http://data.uis. unesco.org/Index.aspx?queryid=172. Zugegriffen: 16. Juni 2020.

Uslucan, H.H., \& Brinkmann, H.U. (Hrsg.). (2013). Dabeisein und dazugehören: Integration in Deutschland. Wiesbaden: Springer.

Van der Zee, K. I., \& Van Oudenhoven, J.P. (2000). The multicultural personality questionnaire: a multidimensional instrument of multicultural effectiveness. European Journal of Personality, 14, 291-309.

Van Dick, R., \& Stegmann, S. (2016). Diversity, Social Identity und Diversitätsüberzeugungen. In Handbuch Diversity Kompetenz (S. 3-15). Wiesbaden: Springer.

Zhang, X., \& Zhou, M. (2019). Interventions to promote learners' intercultural competence: a meta-analysis. International Journal of Intercultural Relations, 71, 31-47.

Zhang, L. (2016). Intercultural competence development: the perceptions of Chinese visiting scholars sojourning abroad. Doctoral dissertation, University of Nottingham.

Publisher's Note Springer Nature remains neutral with regard to jurisdictional claims in published maps and institutional affiliations. 